University of Nebraska - Lincoln

DigitalCommons@University of Nebraska - Lincoln

Faculty Publications from the Department of Engineering Mechanics

Mechanical \& Materials Engineering,

Department of

2010

\title{
Studies of dynamic crack propagation and crack branching with peridynamics
}

Youn Doh Ha Ph.D.

University of Nebraska at Lincoln

Florin Bobaru Ph.D.

University of Nebraska at Lincoln, fbobaru2@unl.edu

Follow this and additional works at: https://digitalcommons.unl.edu/engineeringmechanicsfacpub

Part of the Applied Mechanics Commons, Ceramic Materials Commons, Computational Engineering Commons, Engineering Mechanics Commons, Mechanics of Materials Commons, Structural Engineering Commons, Structural Materials Commons, and the Structures and Materials Commons

Ha, Youn Doh Ph.D. and Bobaru, Florin Ph.D., "Studies of dynamic crack propagation and crack branching with peridynamics" (2010). Faculty Publications from the Department of Engineering Mechanics. 71. https://digitalcommons.unl.edu/engineeringmechanicsfacpub/71

This Article is brought to you for free and open access by the Mechanical \& Materials Engineering, Department of at DigitalCommons@University of Nebraska - Lincoln. It has been accepted for inclusion in Faculty Publications from the Department of Engineering Mechanics by an authorized administrator of DigitalCommons@University of Nebraska - Lincoln. 


\title{
Studies of dynamic crack propagation and crack branching with peridynamics
}

\author{
Youn Doh Ha and Florin Bobaru \\ Department of Engineering Mechanics, University of Nebraska-Lincoln, Lincoln, NE 68588-0526, USA \\ Corresponding author - F. Bobaru, email fbobaru2@unl.edu
}

\begin{abstract}
In this paper we discuss the peridynamic analysis of dynamic crack branching in brittle materials and show results of convergence studies under uniform grid refinement ( $m$-convergence) and under decreasing the peridynamic horizon ( $\delta$-convergence). Comparisons with experimentally obtained values are made for the crack-tip propagation speed with three different peridynamic horizons. We also analyze the influence of the particular shape of the micro-modulus function and of different materials (Duran 50 glass and soda-lime glass) on the crack propagation behavior. We show that the peridynamic solution for this problem captures all the main features, observed experimentally, of dynamic crack propagation and branching, as well as it obtains crack propagation speeds that compare well, qualitatively and quantitatively, with experimental results published in the literature. The branching patterns also correlate remarkably well with tests published in the literature that show several branching levels at higher stress levels reached when the initial notch starts propagating. We notice the strong influence reflecting stress waves from the boundaries have on the shape and structure of the crack paths in dynamic fracture. All these computational solutions are obtained by using the minimum amount of input information: density, elastic stiffness, and constant fracture energy. No special criteria for crack propagation, crack curving, or crack branching are used: dynamic crack propagation is ob-
\end{abstract}

tained here as part of the solution. We conclude that peridynamics is a reliable formulation for modeling dynamic crack propagation.

Keywords: dynamic fracture, crack branching, brittle fracture, peridynamics, nonlocal methods, meshfree methods

\section{Introduction}

\subsection{Literature review of dynamic crack propagation}

In a brittle material, a propagating crack can depart from its original straight trajectory and curve or split into two or more branches. Under very high states of stress, the propagating crack will divide into a river-delta crack pattern (Bowden et al. 1967; Ramulu and Kobayashi 1985). This fragmentation of highly loaded, brittle materials is often a succession of multiple branching of what was initially a single crack. Increases in the roughness of the fracture surface prior to branching were consistently observed in all reported investigations (Ramulu and Kobayashi 1985; Döll 1975). In crack branching of edge notch specimens of brittle materials it has also been observed that the crack tip velocity drops by no more than $5-10 \%$ in the branching region (Döll 1975).

In atomistic models, under conditions that lead to instability of the crack path, cracks can branch without a specific criterion (see Zhou et al. 1996). 
Particle-type models (see Bolander and Saito 1998) are also capable of simulating crack branching. However, none of these methods is able to capture the crack propagation speed or the angle of crack branching correctly. For instance, MD simulations show instabilities that lead, shortly after the bifurcation of a crack, to the propagation of only one of the two branches, the other being arrested. Moreover, the branching angle computed with MD (see Zhou et al. 1996) is greater than $90^{\circ}$, whereas experiments show much smaller crack branching angles (Ramulu and Kobayashi 1985). One may ask whether quantum mechanical calculations are needed to predict the phenomenon of dynamic fracture in brittle materials (see Cox et al. 2005) which is one of the great challenges in dynamic fracture. One likely reason for MD simulations' failure to correctly predict dynamic fracture is that, for example, crack branching events are controlled by the interaction and wave reflections from the boundaries (Ravi-Chandar 1998). Because of this, one would have to either model the entire structure with MD (not a viable option) or use a multiscale model that is capable of transferring the waves between the scales correctly (still an open problem). Numerical simulations based on continuum methods of dynamic crack propagation behavior have been very difficult to develop and, to this date, a reliable method for simulating this complex problem has not been found in spite of considerable efforts in this direction (e.g. Xu and Needleman 1994; Camacho and Ortiz 1996; Ortiz and Pandolfi 1999; Belytschko et al. 2003; Rabczuk and Belytschko 2004; Song et al. 2006). All these methods use some version of cohesive-zone models. As such, they all modify the local continuum mechanics equations and introduce a nonlocal effect given by the parameters and length scales in the cohesive-zone model. To reduce mesh dependency when the grids are refined special methodologies have to be used (Zhou and Molinari 2004). For the existing approaches, the difficulties in modeling dynamic fracture processes like crack branching are many. For example, continuum-type methods using the cohesive FEM or the XFEM require a damage criterion and a tracking of the stresses around the crack tip to decide when to branch the crack. Decisions also have to be taken in terms of the angle of propagation of the branches and about how many branches will be allowed to form. In methods in which the crack advances along the element sides by separating elements from one another, the crack path becomes non-smooth (see $\mathrm{Xu}$ and Needleman 1994; Camacho and Ortiz 1996; Ortiz and Pandolfi 1999). Since the correct path (which minimizes the strain energy) of the crack propagation is not computed correctly, there are significant departures from the true energy released during the crack propagation event. In such cases, reliable prediction of strength of brittle ceramics under impact, for example, becomes difficult. Mesh dependency is an additional problem in cohesive-zone FEM-based methods. Important progress has been recently made by using the XFEM method which allows cracks to pass through the finite elements (see e.g. Belytschko et al. 2003). Subdivision of the cut elements for numerical integration purposes increases the complexity and the cost of the method. This method requires phenomenological damage models and branching criteria, as well as tracking of the crack path using level sets, for example. It is not yet clear if the method is applicable to problems that involve fragmentation and/or multiple crack interactions, branching, and coalescence. The method does not predict the experimentally observed crack propagation speeds (see Song et al. 2008). Cohesivezone based models need to modify the experimental values of the fracture energy by several factors in order to get propagation velocities in the range of measured ones (Song et al. 2008).

In the present contribution we try to answer whether quantum, atomistic, or multiscale models are needed in dynamic fracture in order to correctly simulate the observed crack propagation velocities and crack paths (Cox et al. 2005; Song et al. 2008). We will show that peridynamics is able to correctly model and simulate dynamic fracture, in particular crack branching in brittle materials. Peridynamics, which is a reformulation of continuum mechanics (Silling 2000; Silling et al. 2007b), does not require criteria for crack propagation or crack branching: these happen spontaneously in this method and are autonomously generated by a simple bond-failure criterion that is correlated to the material's energy release rate. The name "peridynamics" comes from the Greek "peri" which means "nearby," and dynamics. Peridynamics is a nonlocal method in which material points interact not only with their nearest neighbors but also with points nearby, inside a horizon. This is what physically happens at the atomic scale, for example, but peridynamics extends this idea to the continuum scale. We will show convergence in terms of the crack path and the crack propagation speed under 
grid refinement (the so-called $\delta$-convergence, Bobaru et al. 2009) and under decreasing peridynamic horizon (the so-called $\delta$-convergence, Bobaru et al. 2009). The crack branching patterns obtained using peridynamics follow remarkably close the experimental results which show secondary branching taking place when higher stress levels are reached at the tip of the pre-notch prior to crack propagation. Moreover, the only input parameters in the model are the Young's modulus, the density, and the fracture energy (which is kept constant, and not a function of the propagation velocity or of the incurred damage, in this first study).

The paper is organized as follows: in the next section we describe the sample problem setup. In Section 2 we briefly review the peridynamic formulation and the connections between the parameters in the formulation and the material properties like the energy release rate. In Section 3 we present the numerical results for the convergence studies. We look at both the crack path and the propagation speed of the crack, as measures of convergence. In Section 4 we analyze the influence of the micromodulus function on crack branching results as well as the solutions for two different brittle materials under higher loading conditions that lead to cascading branching. We also comment on the roughening zones that take place in the branching regions and on the effect of the reflection waves on the propagation paths of the dynamic cracks. The conclusions are given in Section 5.

\subsection{Problem setup}

We consider the following setup as a benchmark problem for analyzing crack branching phenomena: a prenotched thin rectangular plate with $0.1 \mathrm{~m}$ by $0.04 \mathrm{~m}$ as shown in Figure 1 . All simulations in this paper are 2D simulations. For some 3D results we refer the readers to Ha et al. (2010). The materials chosen for this study are selected because for these materials there are experimental results published on the crack propagation velocity in the region of branching or the maximum propagation velocity measured. The two materials used here are a Duran 50 glass (taken form Döll 1975) and a soda-lime glass (taken from Bowden et al. 1967). The material properties are summarized in Table 1. Please note that in the bond-based peridynamic implementation used here, the numerical models will be limited to using a fixed Poisson ratio of $1 / 3$ (for $2 \mathrm{D}$ plane stress problems). If other Poisson ratios are desired, then the state-based

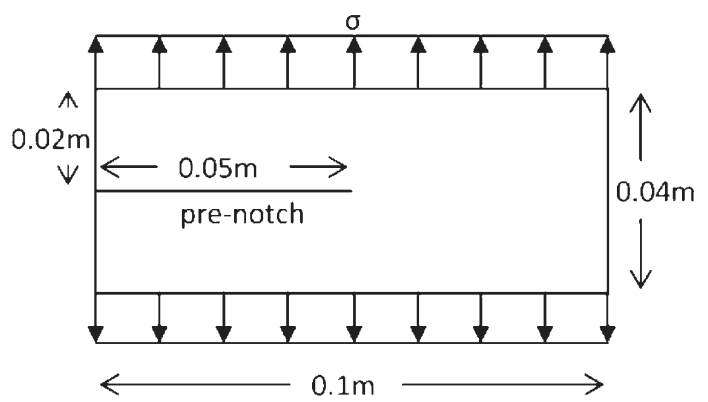

Figure 1. Description of the problem setup for the crack branching study.

peridynamics formulation should be used (see Silling et al. 2007b). For dynamic fracture problems, the Poisson ratio value does not have a significant influence on the propagation speed or the crack path shapes (Silling et al. 2007a).

In the experimental settings the loading of the sample may take tens of seconds or more. In explicit dynamic simulations that would be too expensive to compute. Instead, we choose to apply, along the upper and lower edges (see Figure $1)$, traction loadings $\sigma$ suddenly at the initial time step and maintain this loading constant after that. The theoretical background for the peridynamics analysis is based on Silling's original peridynamics paper (Silling 2000), the imposition of the traction boundary conditions is as in Ha and Bobaru (2009), and the numerical implementation of failure is like in Silling and Askari (2005). The same geometrical setup for studying crack branching simulations has been used in other studies (Belytschko et al. 2003; Rabczuk and Belytschko 2004; Song et al. 2006).

While there is no analytical solution for the crack branching problem, we can compare our simulation results with experiments. Unfortunately, the experimental papers we found do not provide a complete description of the conducted experiment on crack branching: some papers show the crack paths but do not provide crack propagation speed data, others give the propagation speed but do not show the crack paths, and most do not describe in detail the loading conditions. We decided to perform the peridynamic simulations using a setup similar to that used in a few recent simulation papers (Belytschko et al. 2003; Rabczuk and Belytschko 2004; Song et al. 2006). The material parameters, however, are like those used in the experiments (Bowden et al. 1967; Döll 1975). The maximum crack propagation speed, or the 
Table 1. Material properties for Duran 50 and soda-lime glasses Density $(\rho)\left(\mathrm{kg} / \mathrm{m}^{3}\right) \quad$ Young's modulus $(E)(\mathrm{GPa}) \quad$ Poisson ratio $(v)$ Fracture energy $\left(G_{0}\right)\left(\mathrm{J} / \mathrm{m}^{2}\right)$

Duran 50 glass

2,235

Soda-lime glass

2,440
0.2

0.22
204

135 crack propagation speed in the region of branching, is data that is fairly reproducible in experiments and this is reported in Bowden et al. (1967) and Döll (1975), for example. We are not aware of any numerical method that can reproduce the experimentally measured dynamic crack propagation velocity. Note that in previous studies, for certain methods, the fracture energy has to be significantly modified (by several factors) in order to bring the dynamic crack propagation speed closer to the measured values (see, e.g. Belytschko et al. 2003; Rabczuk and Belytschko 2004).

\section{The peridynamic formulation}

The peridynamic formulation (Silling 2000) relies on integration of forces acting on a material point and thus it does not face any of the mathematical inconsistencies seen in the classical continuum mechanics equations. The integration takes place over a "horizon" (which, in principle, extends to infinity but, for convenience is finite) within which the material points are interacting with each other. In certain problems, the size of the horizon can be correlated to an intrinsic material length-scale. However, in many cases a material length scale is not "visible" either because the micro-structure and the specific loading and boundary conditions do not lead to a measurable effect of the length-scale. In such cases, the horizon is selected by the user according to convenience (see Bobaru et al. 2009). Allowing a variable horizon (with a correspondingly scaled micromodulus parameter) defines away of introducing adaptive refinement for this nonlocal method. It is important to notice that peridynamics is a continuum theory, not a particle-type method. This allows the convergence results of the peridynamic solution to the classical elasticity solutions in the limit of the horizon going to zero (Bobaru et al. 2009; Silling and Lehoucq 2008).

An important advantage of peridynamics is the way damage is introduced: material points are connected within the horizon via elastic (linear or nonlinear) bonds that have a critical relative elongation, $s_{0}$, at which they break (Silling 2000).
The critical relative elongation for brittle materials is computed from the experimentally measured value of the fracture energy for a specific material (Silling and Askari 2005). Damage is implemented as the fraction between the number of broken bonds and the number of initial bonds (Silling and Askari 2005). Cracks in peridynamics form as surfaces between material points form, as a consequence of sequential breaking of bonds. Thus, there is no need to track the cracks like in other continuum methods, or to impose criteria for when cracks should branch, change direction, turn, coalesce, etc. Moreover, peridynamics allows for spontaneous generation of cracks where no flaws were present before. This is shown, for example, in Silling et al. (2009) for the crack nucleation and in simulation of spallation (see Xie 2005) where spallation is treated as real fracture and not modeled by void-growth formulations as in existing literature results.

We now briefly review the peridynamic formulation based on Silling's original peridynamics paper (Silling 2000). Also, we consider the summary of the numerical implementation of the traction boundary conditions in peridynamics (Ha and Bobaru 2009) and the formulation for the damage model in peridynamics (Silling and Askari 2005).

The peridynamic equations of motion are given by:

$\rho \ddot{u}(x, t)=\int_{\mathcal{H}} f(u(\hat{x}, t)-u(x, t), \hat{x}-x) d \hat{x}+\boldsymbol{b}(x, t)$

where $f$ is the pairwise force function in the peridynamic bond that connects node $\hat{x}$ to $x$ and $u$ is the displacement vector field. $\rho$ is the density and $\boldsymbol{b}(x, t)$ is the body force. The integral is defined over a region $\mathcal{H}$ called the "horizon," which is the compact supported domain of the pairwise force function around point $x$.

A micro-elastic material (Silling 2000) is defined as one for which the pairwise force derives from a potential $\omega$ :

$f(\eta, \xi)=\frac{\partial \omega(\eta, \xi)}{\partial \eta}$

where $\xi=\hat{x}-x$ is the relative position in the reference configuration and $\boldsymbol{\eta}=\hat{\boldsymbol{u}}-\boldsymbol{u}$ is the relative dis- 
placement. A linear micro-elastic potential is obtained if we take

$\omega(\eta, \xi)=\frac{c(\xi) s^{2} \xi}{2}$

where $\xi=\|\xi\|$ and the relative elongation of a bond is

$s=\frac{\zeta-\xi}{\xi}$

where $\zeta=\|\xi+\eta\|$. The function $c(\xi)$ is called micro-modulus and has the meaning of the bond elastic stiffness. There are various formulations for the micromodulus function and in Section 4.1 we perform tests for dynamic crack propagation to assess the influence of the particular shape of the micro-modulus function on the crack path structure. We will observe that the crack propagation speed is not influenced by the shape of the micro-modulus, once the horizon is reasonably small compared to the dimensions of the structure analyzed. The pairwise force corresponding to a linear microelastic potential has the following form:

$f(\eta, \xi)= \begin{cases}\frac{\xi+\eta}{\|\xi+\eta\|} c(\xi) s, & \xi \leq \delta \\ 0, & \xi>\delta\end{cases}$

where $\delta$ is the radius of the horizon region (which we will also refer to as the horizon). In this paper, we use the constant and conical 2D micro-modulus functions (see Figure 2). Following the same procedure performed to calculate the micro-modulus function in 1D (Bobaru et al. 2009), we obtain the constant micro-modulus function in 2D, plane stress conditions:

$c(\xi)=c_{0}=\frac{6 E}{\pi \delta^{3}(1-v)}$

Similarly, the conical micro-modulus function is obtained as

$c(\xi)=c_{1}\left(1-\frac{\xi}{\delta}\right)=\begin{gathered}24 E \\ \pi \delta^{3}(1-v)\end{gathered}\left(1-\frac{\xi}{\delta}\right)$

The shapes of the constant and conical micromodulus functions are illustrated in Figure 2.

In the bond-based peridynamics, any particle inside the horizon of another particle interacts only through a central potential. This assumption results (for an isotropic, linear, micro-elastic material) in an effective Poisson ratio of $1 / 3$ in $2 \mathrm{D}$ (and $1 / 4$ in 3D), but this limitation is readily eliminated by using the state-based peridynamics (Silling et al. 2007a,b). In this paper, we utilize the bond-
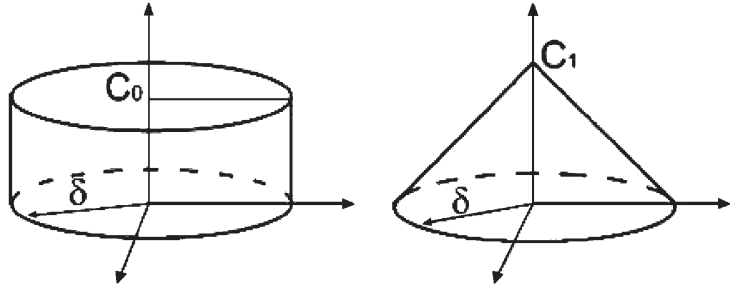

Figure 2. Constant (left) and conical (right) micromodulus functions.

based peridynamics, thus, in all the reported simulations here the effective Poisson ratio is $1 / 3$.

In order to introduce failure into the peridynamic model, we consider that the peridynamic bonds can be broken when they are stretched beyond a predefined limit. We call this limit the "critical relative elongation, $s_{0}$." According to Silling and Askari (2005), there is no force sustained by the bond after its failure. Also, once a bond fails, it is failed forever; this makes the model history dependent. To completely separate a body into two halves across a fracture plane requires breaking all the bonds that initially connected points in the opposite halves (see Silling and Askari 2005). The energy per unit fracture length-(in 2D, fracture area in 3D) for complete separation of the two halves of the body is called fracture energy, $G_{0}$. In 3D, Silling and Askari relate the critical elongation, $s_{0}$, with this measurable quantity $\left(G_{0}\right)$ (Silling and Askari 2005). In 2D with plane stress conditions, the fracture energy can be derived as

$G_{0}=2 \int_{0}^{\delta} \int_{z}^{\delta} \int_{0}^{\cos -1(z / \xi)}\left[\begin{array}{c}c(\xi) s_{0}^{2} \xi \\ 2\end{array}\right] \xi d \theta d \xi d z$

(See Figure 3 for an explanation of this computation.) Substituting the constant micro-modulus function (Equation 6) and rearranging for $s_{0}$, we can rewrite this equation to obtain $s_{0}$

$s_{0}=\sqrt{\frac{4 \pi G_{0}}{9 E \delta}}$

In similar way, the critical relative elongation for the conical micro-modulus function (Equation 7) is

$s_{0}=\sqrt{\frac{5 \pi G_{0}}{9 E \delta}}$

The critical relative elongation depends on the material properties and the horizon $\delta$. Note that as the horizon goes to zero, the critical relative elongation goes to infinity, thus breaking such bonds 


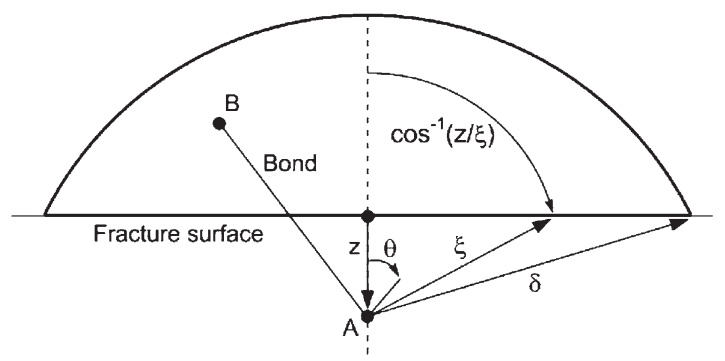

Figure 3. Evaluation of fracture energy. For each point $A$ along the dashed line, $0 \leq \mathrm{z} \leq \delta$, the work required to break the bonds connecting $A$ to each point $B$ in the circular cap is given by Equation (8).

requires larger and larger forces. This agrees with the physical experience at atomic and subatomic scales where, in order to break apart smaller and smaller sized bonds, one needs higher and higher forces. The values for the fracture energy used in this paper are the ones given in Döll (1975) for Duran 50 glass and soda-lime glass materials and measured at the instant of crack branching of a dynamically running crack. Note that there is evidence that the fracture energy varies with the crack propagation speed (see, e.g. Döll 1975). However, other authors point out the fact that the apparent increase in the fracture energy with crack speed may be due to the presence of microcracks (see, e.g. Cox et al. 2005; Ravi-Chandar 1998). For simplicity, in this work, we keep the fracture energy constant and equal to that measured at crack branching. Nevertheless, it is very easy to introduce the velocity-dependent fracture energy (Döll 1975) in our model and we plan to do so in the future. Moreover, it has been observed that peridynamics generates fragment size distributions closer to experimentally measured ones if the critical relative elongation (and therefore the fracture energy) depends on the damage index (ratio of number of broken bonds and number of initial intact bonds) in the following way: if the damage index is larger than some fraction (say 0.2) then the critical relative elongation value increases with the damage index (see Silling 2005). Such damage-dependent model is used in Ha and Bobaru (2010) for dynamic fracture problems. Note that other models have been used in the past to explain dynamic instabilities (see Buehler et al. 2003) in dynamic crack propagation using MD models, but this does not appear to be needed in peridynamics to trigger instabilities or crack branching.

Additionally, since we apply the load abruptly along the upper and bottom boundaries, relatively high tensile stress act along these boundar- ies (see the first figure (a) in Figure 4) at the early stages of the simulation. In order to prevent tearing of the first few layers of nodes from the rest of the plate we set the boundary nodes as no-fail zones. In a no-fail zone the damage index is always zero. The traction boundary conditions are applied to a single layer of nodes at the surface in peridynamics, which is similar to how one imposes these conditions in the FEM, for example. The numerical implementation of traction boundary conditions and the convergence studies are shown in $\mathrm{Ha}$ and Bobaru (2009). These loads are applied suddenly and a shock wave propagates. In Figure 4, we show a few snapshots of the strain energy and close-ups around the tip of the pre-notch of the damage for dynamic crack propagation in the setup shown in Figure 1, for Duran glass. The model has a uniform grid spacing with $\Delta \mathrm{x}=0.125 \mathrm{~mm}$, the horizon $\delta=$ $0.5 \mathrm{~mm}$, and a uniform tensile stress $\sigma=12 \mathrm{MPa}$ is applied. In Figure 4a, the colors denote the magnitude of the elastic strain energy. In Figure 4a note the ripples behind the wave-front caused by the wave dispersion which, in peridynamics, is due to the size of the horizon and the size of the discretization (see discussion of the 1D case in Xie 2005, pp. 40-44). The colors in the right-hand side plots of Figure 4 represent damage levels. Right after the shock wave reaches the center line, the crack starts propagating (compare Figure $4 a, c$ at 6 and $9 \mu$ s).

\section{Numerical studies of convergence in dynamic crack branching}

In peridynamics, we can talk about three types of convergence (see Bobaru et al. 2009 and Figure 5):

- The $\delta$-convergence: $\delta \rightarrow 0$ and $m(=\delta / \Delta \mathbf{x})$ is fixed or increases with decreasing $\delta$ but at a slower rate. In this case the numerical peridynamic approximation converges to an approximation of the classical solution (if this exists), almost everywhere. The larger $m$ is, the closer this approximation becomes. (The convergence is not guaranteed to be uniform in problems with singularities.)

- The $m$-convergence: $\delta$ is fixed and $m \rightarrow \infty$. The numerical peridynamic approximation converges to the exact nonlocal peridynamic solution for the given $\delta$.

- The $(\delta m)$-convergence: $\delta \rightarrow 0$ and $m$ increases with decreasing $\delta$, with $m$ increasing faster than $\delta$ decreases. In this case numerical peridynamic approximation converges to the an- 


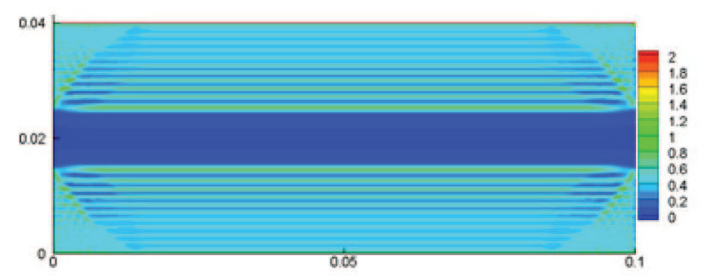

[3 $\mu \mathrm{s}]$

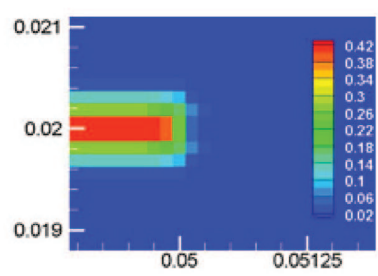

Figure 4. Elastic strain energy and damage map around the precrack tip, at the initial stages of crack propagation $(\delta=0.0005 \mathrm{~m}$,
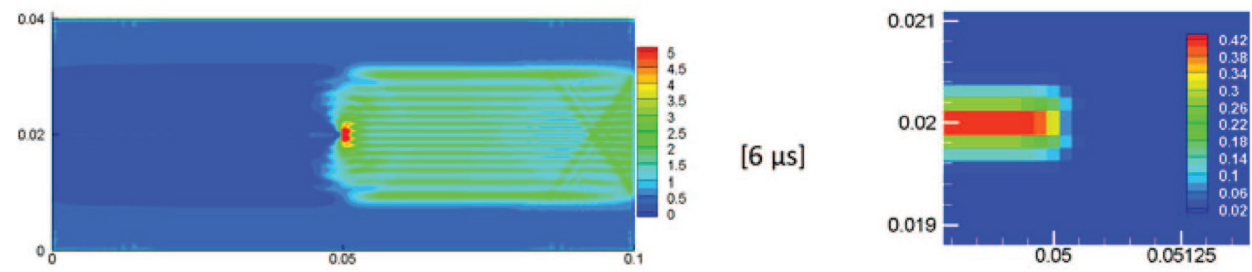
$\Delta \mathrm{x}=0.000125 \mathrm{~m}$, applied load $\sigma=$ 12MPa). a) Elastic [6 $\mu \mathrm{s}]$

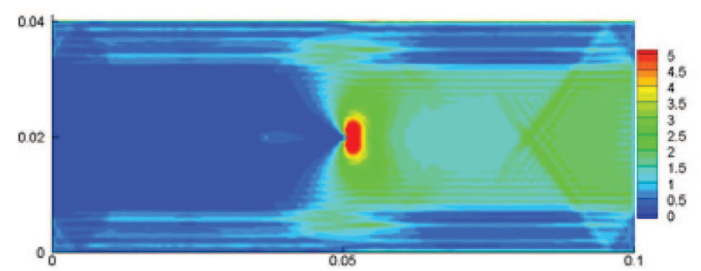

(a)
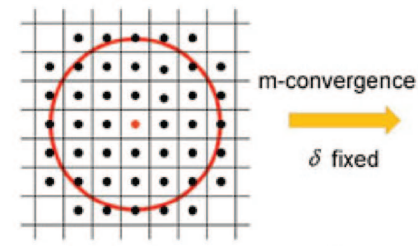

(a)
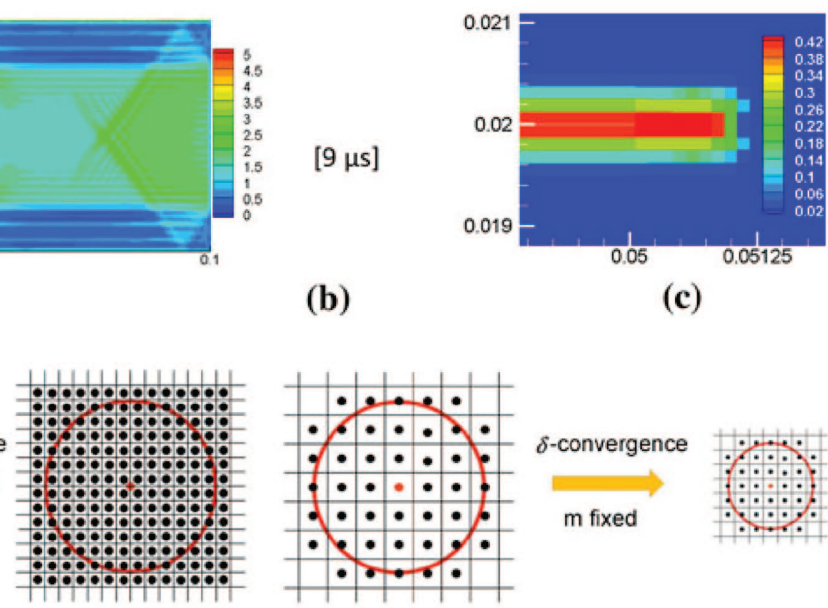

(c) (b)

[9 $\mu \mathrm{s}]$

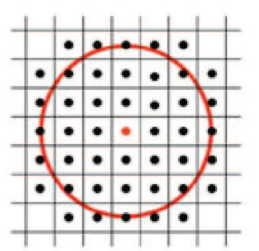
strain energy; $\mathbf{b}$ ) Time; c Crack-tip near-view.

Figure 5. Graphical descriptions for the a) $m$-convergence and $\mathbf{b}$ ) $\delta$-convergence. alytical peridynamic solution and converges uniformly to the local classical solution (if this exists), almost everywhere.

Here we are studying the $m$-convergence and we make some observations related to the $\delta$-convergence for dynamic crack propagation problems. The problem to be analyzed is shown in Figure 1: an edge-notch plate. One way to introduce a pre-crack in peridynamic model is to break all bonds that cross the pre-crack line. Another way is to erase nodes that are along the precrack line in addition to breaking all bonds crossing the lines. The first option, under $m$-convergence, will result in the same "effective" pre-crack. The second option, under $m$-convergence, can also maintain the pre-crack but only if the total volume of the nodes removed remains the same for any grid spacing used. Note, however, that under $\delta$-convergence things are more delicate. The reason is that if we change the horizon the damage area along the pre- crack and in front of the pre-crack tip changes independent of the way in which we introduce the pre-crack in the peridynamic model.

In Figure 6, the initial damage areas of two models with different grid spacings are compared. The color-bar represents damage levels. The thick black line denotes the pre-notch. The triangles and the squares are nodes of the coarse and fine models, respectively. In Figure 6a, the damage area of the coarse model matches with the area of the fine model for the same horizon size of $\delta=2 \mathrm{~mm}$. However, the damage area of the fine model with $\delta=1$ $\mathrm{mm}$ is slightly smaller than the area of the coarse model with $\delta=2 \mathrm{~mm}$, as shown in Figure $6 \mathrm{~b}$.

\subsection{The m-convergence study}

For time integration we use an explicit method, the Velocity-Verlet algorithm. The Velocity-Verlet algorithm (Hairer et al. 2003) is: 


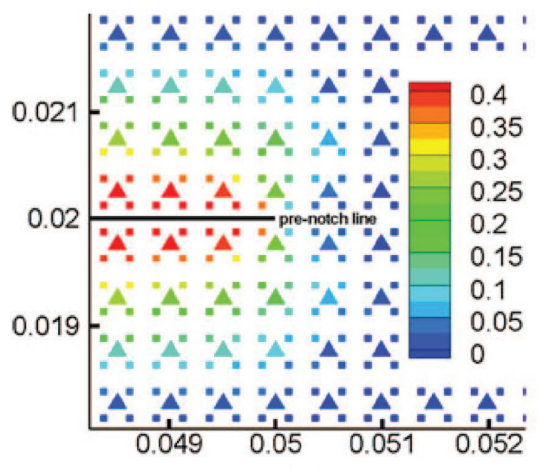

(a)

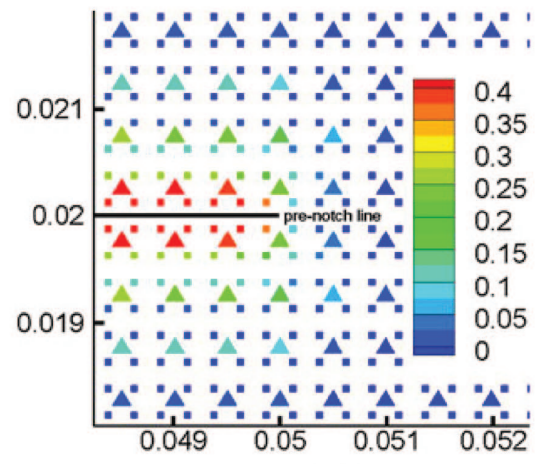

(b)

Figure 6. Relations between the horizon, grid spacing, and the damage area. (triangles = nodes of the coarse model, squares $=$ nodes of the fine model). a) Damage areas on two grids with same horizon $(\delta=0.002 \mathrm{~m}$ for both models); b) Damage areas on two grids with different horizons ( $\delta=0.002 \mathrm{~m}$ for coarse model, $0.001 \mathrm{~m}$ for fine model).

$\dot{u}_{n+1 / 2}=\dot{u}_{n}+\frac{\Delta t}{2} \ddot{u}_{n}$

$\boldsymbol{u}_{n+1}=\boldsymbol{u}_{n}+\Delta t \dot{\boldsymbol{u}}_{n+1 / 2}$

$\dot{u}_{n+1}=\dot{u}_{n+1 / 2}+\frac{\Delta t}{2} \ddot{u}_{n+1}$

where $\boldsymbol{u}, \dot{\boldsymbol{u}}$, and $\ddot{\boldsymbol{u}}$ denote the displacement, velocity, and acceleration vectors, respectively.

We perform the $m$-convergence tests for two different horizon sizes: $\delta=3 \mathrm{~mm}$ and $\delta=2 \mathrm{~mm}$. Please note that these horizon sizes are relatively large compared to the structural dimensions. All models have the uniform grid spacing. A uniform time step size of $25 \mathrm{~ns}$ is used and this is a stable time step for the finest model among all tests performed in this paper, with $\delta=0.5 \mathrm{~mm}$ and $m=4$. A uniform tensile stress $\sigma=12 \mathrm{MPa}$ is applied (as described in the previous section) for all the tests in this section. All computations in this section use the constant micro-modulus function (Equation 6) and the Duran 50 glass material parameters.

We first perform the $m$-convergence study for the fixed horizon $\delta=3 \mathrm{~mm}$. The peridynamic models used for this study are the ones with $\Delta \mathrm{x}=$ $1 \mathrm{~mm}$ (4,326 nodes), $\Delta x=0.5 \mathrm{~mm}$ (16,646 nodes), and $\Delta x=0.25 \mathrm{~mm}(65,448$ nodes $)$. For this test, the model with $\Delta \mathrm{x}=1 \mathrm{~mm}$ has maximum 29 nodes in the horizon $(m=3)$, the model with $\Delta x=0.5 \mathrm{~mm}$ has a maximum of 113 nodes in the horizon $(m=$ 6), and the one with $\Delta x=0.25 \mathrm{~mm}$ has maximum 441 nodes in the horizon $(m=12)$. The crack paths for these cases at $46 \mu$ s are compared in Figure 7. In all damage map plots we use the same range for the color-bar of the damage index as in Figure 6. The results in Figure 7 show that $m$-convergence of the crack path is obtained even for $m$-values as small as 3. In all cases in Figure 7, the crack starts propagating around $7 \mu \mathrm{s}$, and the crack branches around $25 \mu$ s nearby $0.071 \mathrm{~m}$ measured from the left-side of the plate. In these results we notice that a thicker damage zone is produced before the crack branches. This may be an indication of the fracture surface roughness prior to branching that is observed consistently in all reported experimental investigations of crack branching in brittle materials (see, e.g. Ramulu and Kobayashi 1985 and references therein).

For the fixed horizon $\delta=2 \mathrm{~mm}$, the $m$-convergence study is performed using the same three numerical grids as above. Since the horizon is smaller, the corresponding $m$ values will be smaller than previously: the coarsest model with $\Delta \mathrm{x}=1 \mathrm{~mm}$ has maximum 13 nodes in the horizon $(m=2)$, the one with $\Delta x=0.5 \mathrm{~mm}$ has maximum 49 nodes in the horizon $(m=4)$, and the one with $\Delta \mathrm{x}=0.25 \mathrm{~mm}$ has maximum 197 nodes in the horizon $(m=8)$. The crack branching path at $40 \mu$ s for our peridynamic simulations are shown in Figure 8. The crack path given by the coarsest model with $m=2$ is slightly different from the others, but the paths with $m=4$ and 8 are very similar to each other. In Figure 8, crack branching takes place around $24 \mu \mathrm{s}$ and around $0.068 \mathrm{~m}$ from the left edge.

These peridynamic results for two different horizon sizes indicate that $m$-convergence takes place for the dynamic crack branching problem in terms of the crack path and the crack propagation speed, the latter because the crack tip locations at the same times are similar among the different solutions. This is expected to hold for any given $\delta$. It appears that $m=4$ is a good choice because the 


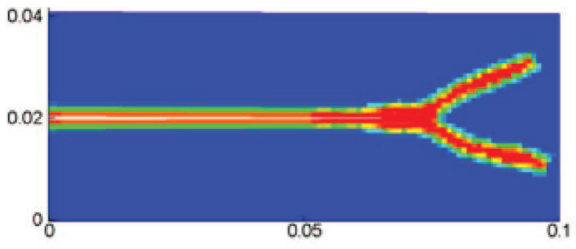

(a)

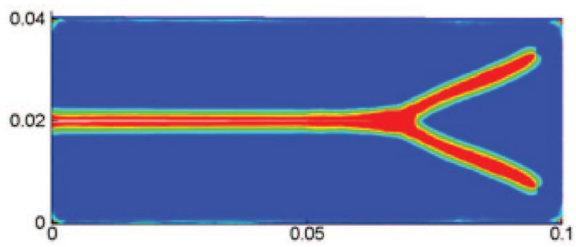

(c)

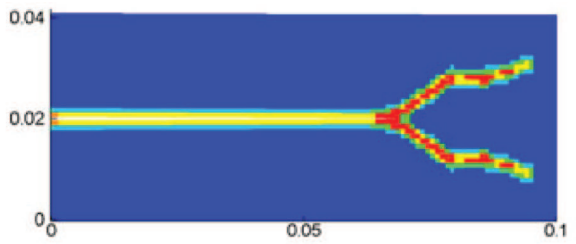

(a)

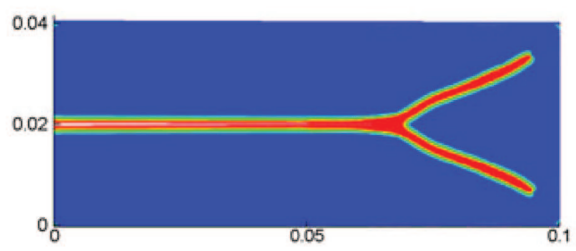

(c)

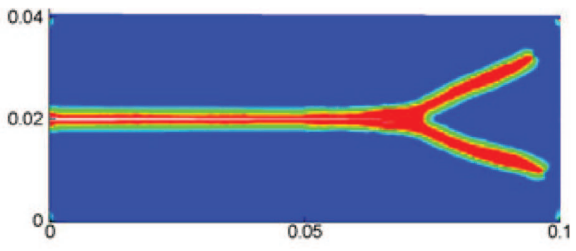

(b)

Figure 7. Crack path computed with different grids for $\delta=0.003$ $\mathrm{m}$ at $46 \mu$ s. a) $m=3, \Delta \mathbf{x}$ $=0.001 \mathrm{~m}$; b) $m=6, \Delta \mathrm{x}$ $=0.0005 \mathrm{~m}$; c) $m=12$, $\Delta \mathrm{x}=0.00025 \mathrm{~m}$.

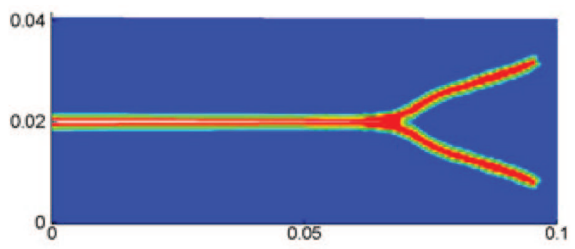

(b)
Figure 8. Crack path computed with different grids for $\delta=0.002$ $\mathrm{m}$ at $46 \mu \mathrm{s}$. a) $m=2$, $\Delta \mathrm{x}=0.001 \mathrm{~m} ; \mathrm{b}) m=4$, $\Delta \mathrm{x}=0.0005 \mathrm{~m} ; \mathrm{c}) \mathrm{m}=$ $8, \Delta \mathrm{x}=0.00025 \mathrm{~m}$. number of nodes inside horizon allows a sufficiently large number of directions along which the true crack path can develop. Using a larger value of $m$ requires higher computational cost, while the results are not affected. In all remaining tests we will use this value of $m$.

\subsection{Crack path under changing horizon $\delta$}

The $\delta$-convergence has to be treated carefully for problems with initial cracks or notches due to the changing size of the initial damage area as discussed in the beginning of Section 3 and Figure 6. The tests in this section are also for the Duran 50 glass material and the constant micro-modulus function (Equation 6).

For a fixed $m=4$, the impact of a changing $\delta$ is investigated by using the four different kinds of horizons, and therefore, four different grids: the coarsest model has $\delta=4 \mathrm{~mm}$ with the uniform grid spacing of $\Delta x=1 \mathrm{~mm}(4,326$ nodes), the subsequent models have half the horizon size of the previous model and half the grid spacing. Thus, the other three models have, respectively, $\delta=2$ $\mathrm{mm}$ and $\Delta \mathrm{x}=0.5 \mathrm{~mm}(16,646$ nodes $), \delta=1 \mathrm{~mm}$ and $\Delta \mathrm{x}=0.25 \mathrm{~mm}(65,448$ nodes $)$, and $\delta=0.5 \mathrm{~mm}$ and $\Delta \mathrm{x}=0.125 \mathrm{~mm}(258,566$ nodes $)$. A uniform time step size of 25 ns (which is a stable time step for the finest model) and the uniform tensile stress $\sigma=$ $12 \mathrm{MPa}$ are applied for all peridynamic models. In all models, the maximum number of nodes in each horizon is 49 . The critical relative elongation $s_{0}$ also changes with a decreasing horizon, see Equation (9). Also, the damage area becomes smaller as the horizon deceases, and this is especially important for the area in front of the pre-crack tip. The $\delta$-convergence, here, has to be understood within this context. Note that there exists the possibility 
that the dynamic fracture behavior is connected to one or multiple length scales (see, e.g. Livne et al. 2007). Material microstructure is likely to influence the dynamic fracture behavior and determine one such physical length scale. The numerical models used in the present paper are for an ideal homogeneous material and the crack propagation is generated by stress waves' interaction due to the shock loading. Notice that crack branching can take place without the influence of the stress wave interaction (see Ravi-Chandar and Knauss 1984a). In the future we also plan to run simulations with quasi-statically applied loads. The results below indicate that, for such homogeneous materials we have $\delta$-convergence in the dynamic crack branching problem. The question of "which material length-scale controls dynamic fracture?" is left for the future since the picture is complicated by how energy is supplied to the region of the crack tip (Ravi-Chandar and Knauss 1984b; Ravi-Chandar 1998). In experiments (e.g. Bowden et al. 1967) it is delivered via quasi-static loading, while in the computations here it is delivered by the stress waves induced through the shock loading.

The crack path at $46 \mu$ s for peridynamic simulations using four uniform grids are shown in Figure 9. Notice that there is a slightly asymmetric path obtained for the case shown in Figure 9c. This is due to the coordinate system used, the $(0,0)$ being at the left bottom corner. When the origin of the coordinate system is moved to the center of the plate, then the symmetry of the solution is recovered. For all horizons the shape of the crack path looks almost identical to one another (see the results in Figure 9). We notice, from monitoring strain energy plots, that the direction of the crack paths after branching is strongly influenced by the reflection elastic waves from the boundaries. Experimental evidence of the influence of the stress wave on the crack path shape is presented in RaviChandar and Knauss (1984b). The effect is that the branching angle of the initial cusp-like shape increases as the branches propagate. We will see that this effect of the elastic waves on the crack path propagation after branching is different for the soda-lime glass material. The reason is that elastic waves propagate faster (due to higher stiffness) and cracks propagate slower in the soda-lime glass compared to the Duran glass.

We look now in more detail at the branching events in the finest model in Figure 9d. Determining where branching occurs can be done in several ways. One way is to consider the time when the right-most nodes with non-zero damage are no longer along the middle line (the direction of the initial crack) but become distributed symmetrically about the mid-line, or the crack direction just before branching. In Figure 10, we show the details of the branching event for the finest model. The branching moment appears to take place between 22.5 and $23 \mu \mathrm{s}$.

\subsection{Comparison of peridynamic crack propagation speed with experimental values}

In this section we compare the crack propagation speed from the peridynamic simulations with those from the experiments in Bowden et al. (1967). We examine the soda-lime glass material with three peridynamic models: a small horizon model with $\delta=0.5 \mathrm{~mm}(258,566$ nodes $)$, a medium horizon model with $\delta=1 \mathrm{~mm}(65,448$ nodes), and a large horizon model with $\delta=2 \mathrm{~mm}$ (16,646 nodes). A uniform tensile stress $\sigma=14 \mathrm{MPa}$ is applied suddenly along the long sides of the plate (see Figure 1). The Velocity-Verlet method is used with a uniform time step size of $25 \mathrm{~ns}$. The constant micromodulus function is used.

The crack paths are very similar for all three models as shown in Figure 11a-c. We compare the crack propagation speeds in Figure 11d. Each point on the crack propagation speed profiles (blue triangles, green squares, and orange circles in Figure 11d) is computed by estimating the location of the crack-tip (after branching we only follow the upper branch) at the time when the datadumps are performed. The data-dumps are done every $2 \mu$ s (or every 80 time-steps) starting from the initial time-step. This implicitly introduces a difference compared to the actual instantaneous crack propagation speed. The crack-tip is determined to be the right-most node which has the damage index larger than 0.35 . In the other words, peridynamic bonds related to this node are broken over 35\% compared to the initial, undamaged state of the node. The crack propagation speed at $t_{\ell}$ is computed by

$V=\frac{\left\|x_{\ell}-x_{\ell-1}\right\|}{t_{\ell}-t_{\ell-1}}$

where $x_{\ell}$ and $x_{\ell-1}$ denote the crack-tip positions at the current time $t_{\ell}$ and at the previous datadump time $t_{\ell-1}$. Here, $\ell=1, \ldots, 81$ corresponding to the total simulation time of $40 \mu \mathrm{s}$. The dotted line shows the maximum fracture speed 1580 $\mathrm{m} / \mathrm{s}$ for the soda-lime glass measured in experi- 


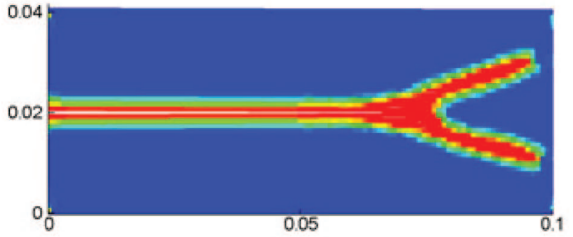

(a)

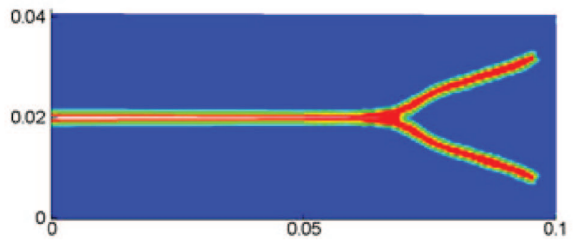

(b)
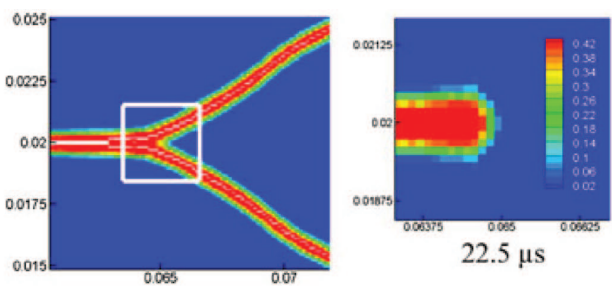

$22.5 \mu \mathrm{s}$

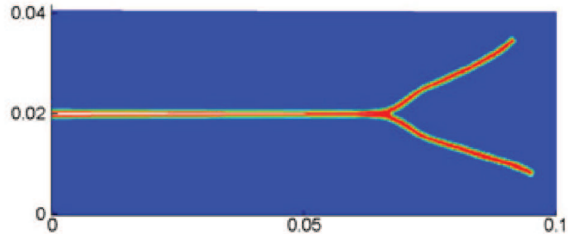

(c)

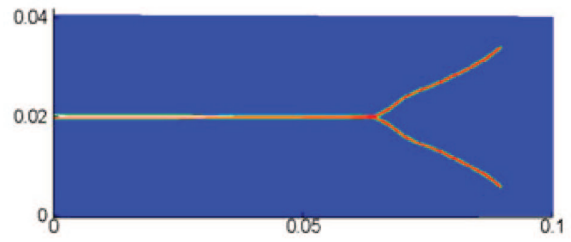

(d)
Figure 9. Crack branching path with various $\delta(m=4)$ using peridynamic analysis at $46 \mu$ s. a) $\delta=$ $0.004 \mathrm{~m}, \Delta \mathrm{x}=0.001 \mathrm{~m}$; b) $\delta=0.002 \mathrm{~m}, \Delta \mathrm{x}=$ $0.0005 \mathrm{~m}$; c) $\delta=0.001$ $\mathrm{m}, \Delta \mathrm{x}=0.00025 \mathrm{~m}$; d) $\delta=0.0005 \mathrm{~m}, \Delta \mathrm{x}=$ $0.000125 \mathrm{~m}$. ments (Bowden et al. 1967) where the loading is, however, quasistatic, in contrast to our dynamically-induced crack propagation. The trends of the peridynamic crack propagation speed results for all models are very similar to one another. This indicates $\delta$ convergence behavior for the crack speed, since the horizon was reduced by half, and the computed cracktip propagation speeds did not change by much. As observed in Figure 11d, the fluctuations for the coarsest model (blue triangles in Figure 11d) are larger than in other fine models (green squares and orange circles in Figure 11d). The main reason for the larger fluctuation is that, for the coarsest model, the crack advances by only a few nodes between the data dumps, while in the finer models the resolution is improved and the number of achievable speed levels is increased.

We next compare the ratios of the numerical and the experimental maximum crack propagation speeds to the Rayleigh wave speed. An approximate expression for the Rayleigh wave speed $c_{R}$ (Graff 1975) is

$\frac{c_{R}}{c_{2}}=\frac{0.87+1.12 v}{1+v}$

where $v$ is the Poisson ratio and the shearwave speed $c_{2}$ is given by $c_{2}=(\mu / \rho)^{1 / 2}$. We select the maximum computed crack speed value at $24 \mu$ s from the numerical result with the smallest horizon model (see Figure 11d). In Table 2, the ratio of this numerical maximum propagation speed to the Rayleigh wave speed $c_{R}$ is compared with the ratio of the experimentally measured maximum fracture speed (Bowden et al. 1967) for the soda-lime glass material.

The observation in Section 4.2 about how wave reflections from the boundaries affect the crack propagation path also explains why the branching angles seen in Bowden et al. (1967) are different from the ones we obtained here (for the soda-lime glass). The geometry used here is different from the one used in Bowden et al. (1967), where the notch is a short one, running parallel to the short side of the plate, the side boundaries are farther away than in our case where the notch is long and parallel to the long side of the plate. The different geometry will strongly influence the propagation speed in the dynamic loading case since the stress waves are supplying energy in the fracture region and that has been observed to influence both the shape of the crack path and the crack propagation speed (Ravi-Chandar and Knauss 1984b).

The results obtained with the finest horizon (and grid spacing) give a maximum crack propagation speed value that is about $6 \%$ larger than the experimentally measured maximum crack speed for sodalime glass of $1580 \mathrm{~m} / \mathrm{s}$ (see Bowden et al. 1967). These encouraging results, however, have to be un- 


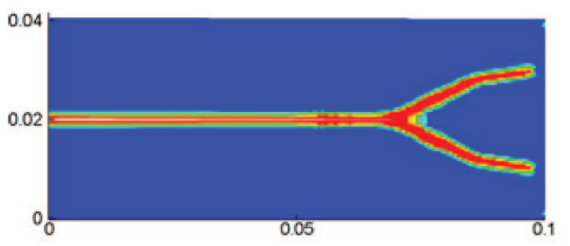

(a)

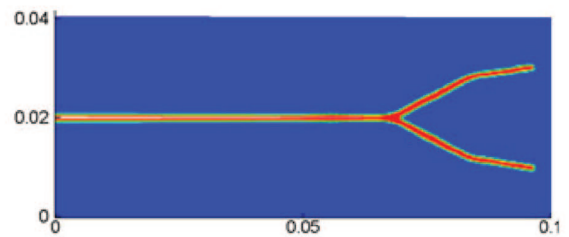

(b)

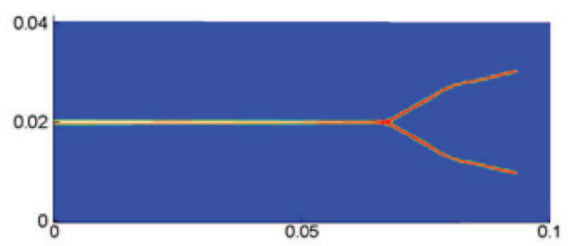

(c)

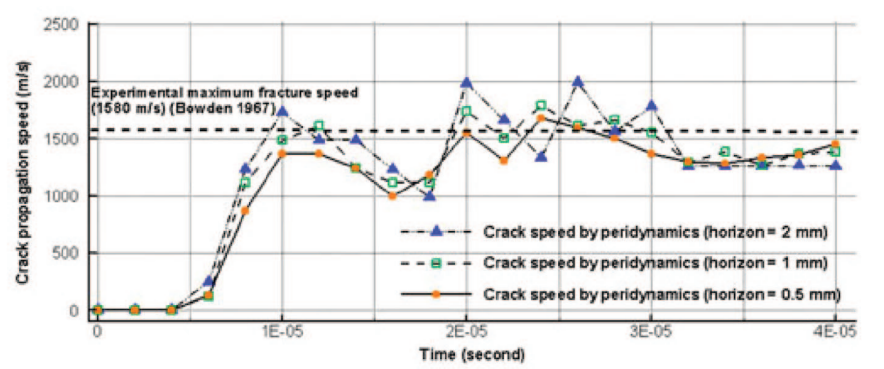

(d)

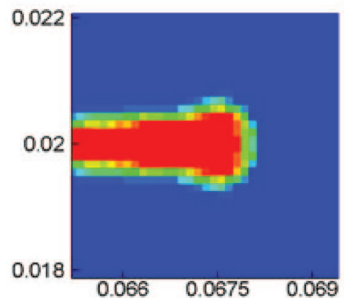

(e)

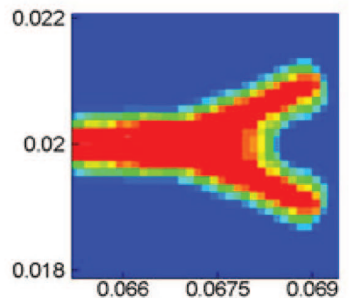

(f)
Figure 11. Crack paths and crack propagation speeds for soda-lime glass (for $\delta$-convergence with $m$ $=4$ ).

a) Crack path at $40 \mu \mathrm{s}$ $(\delta=0.002 \mathrm{~m})$;

b) Crack path at $40 \mu \mathrm{s}$ $(\delta=0.001 \mathrm{~m})$;

c) Crack path at $40 \mu \mathrm{s}$ $(\delta=0.0005 \mathrm{~m})$;

d) Crack propagation speed for three different horizons;

e) Close-up at $20.5 \mu \mathrm{s}$ for the finest horizon; f) Close-up at $21.5 \mu \mathrm{s}$ for the finest horizon.

Table 2. The ratios of numerically computed and experimental maximum fracture speeds to the Rayleigh wave speed

\begin{tabular}{lcc}
\hline & Fracture speed, $V(\mathrm{~m} / \mathrm{s})$ & $V / c_{R}$ \\
\hline Peridynamics & 1679 & 0.53 \\
Experiment (Bowden et al. 1967) & 1580 & 0.50 \\
\hline
\end{tabular}

derstood in context of the different type of loading conditions used in the computations compared with the setup in Bowden et al. (1967). Further comments and analysis on this subject are given in $\mathrm{Ha}$ et al. (2010). We also note that here we use a constant fracture energy model. This may not be the case in reality where the critical fracture energy can change with, for example, the speed of crack propagation. In Döll (1975), for instance, the fracture en- ergy is measured to depend on the propagation velocity. This issue of the dependence of the fracture energy on the crack propagation speed and/or the local damage is also discussed, from the point of view of experiments, in the more recent review article (Ravi-Chandar 1998). In Ha and Bobaru (2010), we report on results that also use a modified critical relative elongation for damaged nodes so that nodes with damage levels over a certain value have 
a larger critical relative elongation. This, in effect, implements a change in the critical fracture energy depending on the damage level at a particular point in the material. The influence of using a damagedependent critical relative elongation on the crack propagation speed is discussed in Ha and Bobaru (2010). This modification has been observed (Silling 2005) to give better results in fragmentation problems. Without the damage-dependent critical elongation model, peridynamics would create a lot of smaller fragments, while using this modification, the number and size distribution of fragments match experimental observations.

We also note that the crack speed profiles have a very similar pattern with the experimental speed profile shown in Figure 11 in reference (Field 1971).We now quote from page 19 in Field 1971 which describes the experimental observations of crack branching:

... The transition region and branch occur when the crack has reached a high proportion of its maximum velocity. The first serious roughening of the fracture surface gave a slight, but detectable, slowing of the crack. Following crack bifurcation the surfaces of the two new cracks normally appear mirror smooth, indicating a somewhat lower velocity than in the transition region. However, the branching does not cause the fracture front velocity to drop to zero or even near it. This is demonstrated by the fracture of toughened glass where the velocity of the front progresses at a relatively uniform velocity of nearly $1,500 \mathrm{~m} / \mathrm{s} .$.

The peridynamic results in Figure 11 match surprisingly well with each and every observation in the quoted text above. Small fluctuations of the speed profile, and a slowing of the crack, appear around 14-16 $\mu$ s (see Figure 11d), which is similar to what happens during the "initial roughening" in Figure 11 in Field (1971). Please note that our model is too coarse to capture actual roughening of the crack surfaces. Indirect evidence of roughness, however, may be observed from our computations, and this is discussed next. The more severe roughening discussed in Field (1971) is seen in Figure 11e, f, as a wider damage zone. This happens just before branching of the crack, the same as in the experiments. At that stage, the crack has been moving at a high proportion of its maximum velocity. After branching, we observe a small drop in the propagation velocity, and the damage region along the crack paths is indicative of smooth crack surfaces of each branch (see Figure 11f).
It is interesting to note the correlation between the stress waves that continue to propagate and reflect from the boundaries and the crack propagation speed. The more recent experimental evidence points towards this interaction as one main cause of crack branching (see Ravi-Chandar 1998; Ravi-Chandar and Knauss 1984b). Our peridynamic simulations confirm this point of view. It appears that the speeding, slowing down, speeding, and then slowing slightly in the region of branching, of the crack tip during the time interval from 5 to $20 \mu$ s is directly caused by the way the elastic strain energy concentrates towards (which results in speeding of the crack tip) or disperses away (which results in slowing down of the crack tip) from the front of the crack path. This is easier to see in a movie of the dynamic crack propagation process (Ha et al. 2010).

4 Numerical results for different micro-modulus functions and different materials at higher stress levels

\subsection{Constant versus conical micro-modulus functions}

In the following we compare the results for two different micro-modulus functions. The tests are performed using the soda-lime glass material properties, and the numerical model is the same except for the type of micro-modulus functions: the constant micro-modulus (Equation 6) and the conical micro-modulus function (Equation 7).

The peridynamic models have $\delta=1 \mathrm{~mm}$ and grid spacing $\Delta x=0.25 \mathrm{~mm}(65,448$ nodes). The Velocity- Verlet algorithm is used for the time integration with a uniform time step size of $25 \mathrm{~ns}$. The uniform tensile stress $\sigma=10 \mathrm{MPa}$ is applied for the model in Figure 1. In Figure 12 we compare the crack branching paths at $50 \mu$ s for the constant and conical micro-modulus functions. We observe very similar branching paths up to a point when, due to the different reflections waves produced, the branches in the conical micromodulus case splay out more than in the constant micro-modulus case. Thus, the particular shape of the micro-modulus function influences the crack propagation path, but to a small extent. In Xie (2005), it was shown that a flux-corrected transport (FCT) algorithm can eliminate the ripples behind a shock wave in a peridynamics simulation. The FCT algorithm is not used in this paper and thus the ripples behind the shock waves are not eliminated. Since 


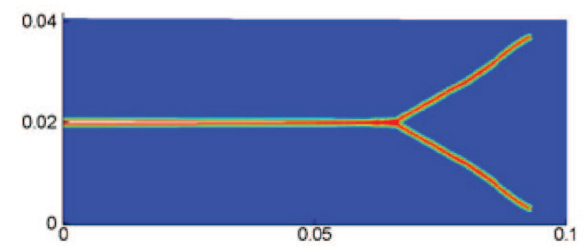

(a)

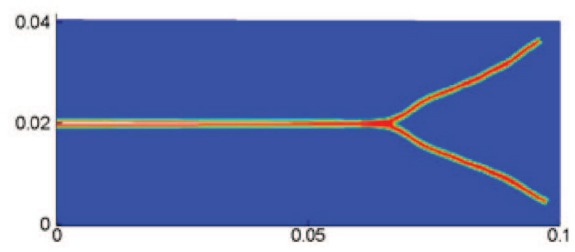

(b)

Figure 12. Crack paths for different micro-modulus functions at $50 \mu \mathrm{s}(\delta=0.001 \mathrm{~m}, \Delta \mathrm{x}=0.00025 \mathrm{~m})$ for sodalime glass. a) Solution with conical micro-modulus; b) Solution with constant micro-modulus.

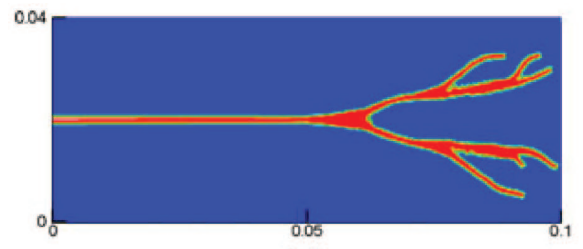

(a)

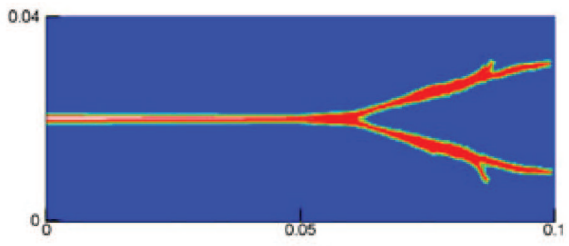

(b)

Figure 13. Crack paths for two different materials under higher loading conditions $(\delta=0.001 \mathrm{~m}, \Delta \mathrm{x}=0.00025$ m). a) Damage map for soda-lime glass at $28 \mu \mathrm{s}$; b) Damage map for Duran 50 glass at $32 \mu \mathrm{s}$.

a different micro-modulus function creates different dispersion curves (see Silling 2000), the oscillations behind the shock wave in our computations (see Figure 4) interfere with the crack path and create differences in the propagation directions between the two models. Note, however, that the crack propagation speeds for the two different cases are very close to one another.

\subsection{Crack branching patterns for two different ma- terials under higher stress levels}

We compare the crack branching patterns between the soda-lime glass and Duran 50 glass under higher loading conditions. The peridynamic models for both materials have $\delta=1 \mathrm{~mm}$ and grid spacing $\Delta x=0.25 \mathrm{~mm}(65,448$ nodes). The VelocityVerlet algorithm with a uniform time step size of 25 ns is employed, and the uniform tensile stress $\sigma=$ $24 \mathrm{MPa}$ is applied suddenly at the initial time-step. In Figure 13, we observe that cascading branching takes place for the soda-lime material, while for the Duran glass, under these particular conditions, the branching events attempted after the main one are arrested. We also note the curving of the secondary branches for the soda-lime case. Experimental confirmation of this phenomenon is given in RaviChandar and Knauss (1984b), for example. The reason for both the arrested branches and the crack path curving rests with the particular way the stress waves are moving through each material and reflecting from the boundaries.

A close examination of the strain energy maps during the crack propagation process clearly shows how the strain elastic energy concentrates in certain regions and the particular incident angle at which the elastic energy waves meet the crack tip can result in bending the crack path for the soda-lime case (see Figure 14a) or in arresting the propagation of secondary branches for the Duran glass case (see Figure 14b). In Figure 14, the elastic strain energy is plotted on the top row figures for areas around the front of the crack propagation path at two different times (21 and $22 \mu$ s for soda-lime glass and 24 and $26 \mu$ s for Duran glass). The bottom row of figures in Figure 14, shows the damage index at similar time steps and at various degrees of close-ups around the top-most branches. For the soda-lime glass (Figure 14a), the reflection waves hit the crack tip of the second branching event at an inclined angle (see strain energy at $21 \mu \mathrm{s})$. This bends the topmost branch as seen from the sequence of plots at 23,25 , and $27 \mu \mathrm{s}$, and in Figure 13a at $28 \mu \mathrm{s}$. For the Duran glass case (Figure 14b), the reflection waves meet the crack tip at an angle that suppresses, or arrests, the top-most branch and its symmetrical, lower-most branch.

We remark that in the soda-lime case, the conditions allow for yet another branching event from some of the secondary crack paths. Also, the thicker 


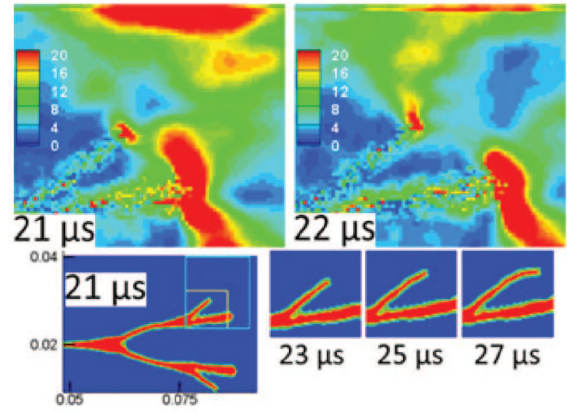

(a)

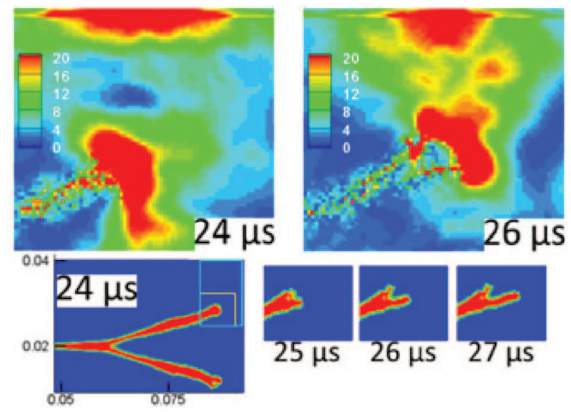

(b)
Figure 14. Elastic strain energy around the crack tip areas (top row of figures) and damage maps (bottom row of plots) at times corresponding to the secondary branching events. Left plots are for soda-lime glass, right plots are for Duran glass. a) Soda-lime glass; b) Duran 50 glass. damage zone following the primary branching event for the Duran glass indicates a rough zone where conditions where close to those that would produce branching. The particular interaction of the crack path with the reflection stress waves arrested that branching event before it happened. Later on, around $26 \mu \mathrm{s}$, secondary branching happens but some of the branches are arrested soon after forming, while the others continue to propagate (see Figure 13b).

Note that here, as before, we used a constant fracture energy that corresponds to that measured at branching and reported in Döll (1975). A discussion about the symmetry and the symmetry breaking in the peridynamic simulations is included in Ha and Bobaru (2010).

\section{Conclusions}

In this paper we presented some detailed studies of modeling dynamic fracture and capturing crack branching events in brittle materials using peridynamics. The results demonstrate that dynamic fracture phenomena are captured by the peridynamic formulation very well. All the details of dynamic crack branching in brittle materials reported in the experimental literature are recovered by our peridynamic simulations, naturally and without having to insert various special crack propagation criteria, for example, for when and how branching should take place. In both the experiments and the peridynamic model, branching occurs in a region where the crack propagation speed reaches a high proportion of its maximum value. There is roughening before branching in the experiments and that is captured as a thicker damage zone ahead of the branching region by the peridynamic simulations. There is a small, but detectable, slowing of the crack propagation speed after branching in both the experiments and our computational results. There is cascade branching in the experiments when higher stress levels are attained before the crack starts to propagate, and we also observed that in our peridynamic solutions when we increased the magnitude of the applied loads.

Convergence in terms of the number of nodes covered by the peridynamic horizon is obtained, and the crack path and crack propagation speed stabilize, or converge, once the horizon becomes of sub-millimeter size, for the sample that measured in centimeters. A small influence of the specific shape of the peridynamic micro-modulus function is observed on the crack propagation path but not on the propagation speed.

Our results for this complex physical process shed light over the question of why Molecular Dynamics (MD) simulations fail to correctly predict crack branching: the phenomenon involves scales of the size of the entire structure since it is the propagation of the elastic strain energy (stress waves) and their reflection from the boundaries of the structure that control the crack propagation process (in terms of the propagation speed and crack path direction) in dynamic fracture. We also note the correlation between the size of the horizon at which the peridynamic results appear to no longer change and the "characteristic interaction distance" talked about in Streit and Finnie (1980), Ramulu and Kobayashi (1985). This issue requires further investigation which we plan for the future.

The overall trend of the crack propagation speed from our simulations showed a remarkable resemblance to the experimental speed profiles reported by others. We compared the maximum crack propagation speed obtained with peridynamics with that from the experiments and the value, for the 0.5 $\mathrm{mm}$ horizon case, was about $6 \%$ larger than the experimental value for soda-lime glass. While the loading conditions are different (quasi-static in experiments, dynamic in our simulations), the result 
is remarkable given that the input in our model was a constant value of the critical fracture energy equal to that measured at crack branching. In conclusion, peridynamics succeeds in correctly modeling crack branching in brittle plates, one of the main open problems in modeling dynamic fracture.

\section{Acknowledgments}

The authors would like to acknowledge the financial support from the Boeing Company (project manager: Dr. E. Askari) and from Sandia National Laboratories (project manager: Dr. S. A. Silling) without which this work would not have been possible. Very useful discussions with Dr. M. L. Parks and Dr. S. A. Silling regarding time integration algorithms are also gratefully acknowledged. The authors would also like to thank the two anonymous reviewers for International Journal of Fracture for their insightful comments. The computations in this paper have been completed using facilities at the University of Nebraska's Holland Computing Center.

\section{References}

Belytschko T, Chen H, Xu J, Zi G (2003) Dynamic crack propagation based on loss of hyperbolicity and a new discontinuous enrichment. Int J Numer Methods Eng 58:1873-1905

Bobaru F, Yang M, Alves LF, Silling SA, Askari E, Xu J (2009) Convergence, adaptive refinement, and scaling in 1D peridynamics. Int J Numer Methods Eng 77:852-877

Bolander JE Jr, Saito S (1998) Fracture analyses using spring networks with random geometry. Eng Fract Mech 61:569- 591

Bowden FP, Brunton JH, Field JE, Heyes AD (1967) Controlled fracture of brittle solids and interruption of electrical current. Nature 216:38-42

Buehler MJ, Abraham FF, Gao H (2003) Hyperelasticity governs dynamic fracture at a critical length scale. Nature 426:141-146

Camacho GT, Ortiz M (1996) Computational modelling of impact damage in brittle materials. Int J Solids Struct 33:2899-2938

Cox BN, Gao H, Gross D, Rittel D (2005) Review: modern topics and challenges in dynamic fracture. J Mech Phys Solids 53:565-596

Döll W (1975) Investigations of the crack branching energy. Int J Fract 11:184-186

Field JE (1971) Brittle fracture: its study and application. Contemp Phys 12(1):1-31

Graff KF (1975) Wave motion in elastic solids. Dover, New York

Ha YD, Bobaru F (2009) Traction boundary conditions in peridynamics: a convergence study. Technical report, Department of Engineering Mechanics, University of Nebraska-Lincoln, Lincoln, Nebraska

Ha YD, Bobaru F (2010) The effect of damage-dependent fracture energy on symmetry-breaking in dynamic brittle fracture. Technical report, Department of Engineering Mechanics, University of Nebraska-Lincoln, Lincoln, Nebraska.

Ha YD, Bobaru F, Parks ML (2010) Dynamic brittle fracture: crack branching predictions with peridynamics (submitted)
Hairer E, Lubich C, Wanner G (2003) Geometric numerical integration illustrated by the Störmer/Verlet method. Acta Numer, 1-51

Livne A, Ben-David O, Fineberg J (2007) Oscillations in rapid fracture. Phys Rev Lett 98(12):124301

Ortiz M, Pandolfi A (1999) Finite-deformation irreversible cohesive elements for three-dimensional crack-propagation analysis. Int J Numer Methods Eng 44:1267-1282

Rabczuk T, Belytschko T (2004) Cracking particles: a simplified meshfree method for arbitrary evolving cracks. Int J Numer Methods Eng 61:2316-2343

Ramulu M, Kobayashi AS (1985) Mechanics of crack curving and branching-A dynamic fracture analysis. Int J Fract 27:187-201

Ravi-Chandar K (1998) Dynamic fracture of nominally brittle materials. Int J Fract 90:83-102

Ravi-Chandar K, Knauss WG (1984a) An experimental investigation into dynamic fracture: III. On steadystate crack propagation and crack branching. Int J Fract 26:141-154

Ravi-Chandar K, Knauss WG (1984b) An experimental investigation into dynamic fracture: IV. On the interaction of stress waves with propagating cracks. Int J Fract 26:189-200

Silling SA (2000) Reformulation of elasticity theory for discontinuities and long-range forces. J Mech Phys Solids 48:175- 209

Silling SA (2005) Fragmentation modeling with EMU. Technical report, Computational Physics Department, Sandia National Laboratories, Albuquerque, New Mexico

Silling SA, Askari E (2005) A meshfree method based on the peridynamic model of solid mechanics. Comput Struct 83:1526-2535

Silling SA, Demmie P, Warren TL (2007a) Peridynamic simulation of high-rate material failure, 2007 ASME applied mechanics and materials conference, Austin, TX, June 6

Silling SA, Epton M, Weckner O, Xu J, Askari E (2007b) Peridynamic states and constitutive modeling. J Elast 88:151-184

Silling SA, Lehoucq RB (2008) Convergence of peridynamics to classical elasticity theory. J Elast 93:13-37

Silling SA, Weckner O, Askari E, Bobaru F (2010) Crack nucleation in a peridynamic solid. Int J Fract 162:219-227

Song J, Areias PMA, Belytschko T (2006) A method for dynamic crack and shear band propagation with phantom nodes. Int J Numer Methods Eng 67:868-893

Song JH, Wang H, Belytschko T (2008) A comparative study on finite element methods for dynamic fracture. Comput Mech 42:239-250

Streit R, Finnie I (1980) An experimental investigation of crack-path directional stability. Exp Mech 20:17-23

Xie W (2005) Peridynamic flux-corrected transport algorithm for shockwave studies. Master thesis, Department of Engineering Mechanics, University of Nebraska-Lincoln, Lincoln, Nebraska

Xu XP, Needleman A (1994) Numerical simulations of fast crack growth in brittle solids. J Mech Phys Solids 42:1397- 1434

Zhou F, Molinari JF (2004) Dynamic crack propagation with cohesive elements: a methodology to address mesh dependency. Int J Numer Methods Eng 59:1-24

Zhou SJ, Lomdahl PS, Thomson R, Holian BL (1996) Dynamic crack processes via molecular dynamics. Phys Rev Lett 76(13):2318-2321 